\title{
Quantum phase transition of ultracold bosons in the presence of a non-Abelian synthetic gauge field
}

\author{
T. Graß, ${ }^{1}$ K. Saha, ${ }^{2}$ K. Sengupta, ${ }^{2}$ and M. Lewenstein ${ }^{1,3}$ \\ ${ }^{1}$ ICFO-Institut de Ciències Fotòniques, Mediterranean Technology Park, 08860 Castelldefels (Barcelona), Spain \\ ${ }^{2}$ Theoretical Physics Department, Indian Association for the Cultivation of Science, Kolkata-700032, India. \\ ${ }^{3}$ ICREA-Institució Catalana de Recerca i Estudis Avançats, Lluis Companys 23, 08010 Barcelona, Spain
}

(Dated: October 13, 2018)

\begin{abstract}
We study the Mott phases and the superfluid-insulator transition of two-component ultracold bosons on a square optical lattice in the presence of a non-Abelian synthetic gauge field, which renders a $S U(2)$ hopping matrix for the bosons. Using a resummed hopping expansion, we calculate the excitation spectra in the Mott insulating phases and demonstrate that the superfluid-insulator phase boundary displays a non-monotonic dependence on the gauge field strength. We also compute the momentum distribution of the bosons in the presence of the non-Abelian field and show that they develop peaks at non-zero momenta as the superfluid-insulator transition point is approached from the Mott side. Finally, we study the superfluid phases near the transition and discuss the induced spatial pattern of the superfluid density due to the presence of the non-Abelian gauge potential.
\end{abstract}

\section{INTRODUCTION}

Experimental systems involving ultracold bosons in optical lattices provide us with a unique test bed for studying quantum phase transitions [1, 2] and for mimicking strongly correlated condensed matter systems [3]. It is well-known that the simplest of such systems with bosonic ultracold atoms are well represented by the BoseHubbard $(\mathrm{BH})$ model for which the superfluid-insulator transition has been studied both theoretically 44 9] and experimentally [1, 10. These ideas have also been extended to systems of multi-species and higher spin bosons which provide richer phase diagrams [11-13]. Such theoretical works have been supported by recent experiments involving studies of both equilibrium phase transitions and closed non-equilibrium dynamics in some of these systems 14, 15.

More recently, there have been several theoretical proposals and concrete experimental realization of synthetic Abelian vector potentials for ultracold gases by subjecting them to carefully tuned Raman lasers (for a review see Ref. 16). These lasers induce a space or time dependent shift in the energy dispersion of the atoms at a given momenta and thus emulate the effect of a synthetic magnetic/electric field $17+23$. It has been shown that spatially varying Abelian gauge potentials acting on a bosonic gas within an optical lattice have a profound effect on both the superfluid-insulator transition and the superfluid (SF) state to which the transition takes place [24 26]. In particular, the positions of the precursor Mott peaks shift to finite momenta and the SF density develops a spatial ordering pattern which can be detected in standard experiments. Similar, albeit more complicated, theoretical proposals have been put forward for realization of synthetic $S U(2)$ gauge potentials for neutral atoms 27 29. However, the effect of non-Abelian gauge potentials on the superfluid-insulator transition of ultracold bosons has not been studied so far.

In this paper we consider a two-species boson sys- tem described by a $\mathrm{BH}$ model in a square optical lattice and study the effect of a $S U(2)$ gauge potential on its superfluid-insulator transition. Our work involves an extension of the resummed hopping expansion in Ref. 30 and 31 and is somewhat similar to the strong coupling expansion technique of Ref. 7. Here these methods are applied to the case of two-species bosons in the presence of an $S U(2)$ gauge field. The main results of this work are the following. First, we show that even for the simplest non-Abelian gauge field, described by a constant $S U(2)$ gauge potential, the interplay of interand intra-species interactions and the presence of the external gauge field leads to qualitative changes in several aspects of the superfluid-insulator transition. Second, we compute the momentum distribution of the bosons in the Mott phase near the superfluid-insulator transition and show that the precursor peaks occur at finite, rather than zero, momenta due to the presence of the gauge field. We find a sudden change of the peak positions when the strength of the gauge field is varied in certain parameter regimes. This indicates that a slight change in the gauge field strength has strong impact on the dynamical behavior of the system and might be especially relevant for its non-equilibrium dynamics. This behavior is somewhat reminiscent of the QPT of excited states discussed in Ref. 32 and of the abrupt sign change of the Hall conductivity which has recently been found for a system of hardcore bosons 33. Third, we demonstrate that the superfluid-insulator phase boundary displays a non-monotonic dependence on the strength of the gauge field leading to re-entrant superfluid-insulator transitions with the variation of the strength of the gauge field for a fixed hopping strength. Finally, we construct an effective Landau-Ginzburg theory for the superfluid-insulator transition and use it to chart out the nature of the SF phase into which the transition takes place. We show that for a constant non-Abelian gauge field, the SF density near the transition does not exhibit any spatial ordering. This feature is to be contrasted with the case where an 
Abelian flux (with half flux quanta per lattice plaquette) is added over the existing $S U(2)$ potential leading to a spatial pattern in the SF density.

The organization of the rest of the paper is as follows. We outline the model describing our system in Section II The Mott insulating (MI) phase is analyzed in Section III. This is followed by the analysis of the SF phase in Section IV. Finally, we conclude in Sec. V.

\section{MODEL}

It is well-known that a $S U(2)$ gauge field can be generated for a system of two-species ultracold bosons by distinguishing between the atoms in two different Zeeman levels representing the two 'flavors' of the non-Abelian theory [27. In doing so, one substitutes the standard hopping process by a laser-assisted tunneling, which may depend on the position and the state of the atom. One of the crucial features of such a tunneling is its ability to flip the state of the atom. Taking into account local interactions between the atoms, the effective Hamiltonian describing the system can be seen to be the same as that of a two-species BH model [11, 12, 34, with an additional non-Abelian vector potential in the hopping term providing the additional inter-species coupling. Defining the number operators $\hat{\mathrm{n}}_{i}^{\mathrm{a}}=\hat{\mathrm{a}}^{\dagger}{ }_{i} \hat{\mathrm{a}}_{i}$ and $\hat{\mathrm{n}}_{i}^{\mathrm{b}}=\hat{\mathrm{b}}_{i}^{\dagger} \hat{\mathrm{b}}_{i}$, where $\hat{\mathrm{a}}_{i}$ and $\hat{\mathrm{b}}_{i}$ denote the boson annihilation operators of the two species, the local part of the Hamiltonian reads

$$
\begin{gathered}
\hat{\mathrm{H}}_{0}=\sum_{i}\left[\frac{U^{\mathrm{aa}}}{2} \hat{\mathrm{n}}_{i}^{\mathrm{a}}\left(\hat{\mathrm{n}}_{i}^{\mathrm{a}}-1\right)+\frac{U^{\mathrm{bb}}}{2} \hat{\mathrm{n}}_{i}^{\mathrm{b}}\left(\hat{\mathrm{n}}_{i}^{\mathrm{b}}-1\right)\right. \\
\left.+U^{\mathrm{ab}} \hat{\mathrm{n}}_{i}^{\mathrm{a}} \hat{\mathrm{n}}_{i}^{\mathrm{b}}-\mu^{\mathrm{a}} \hat{\mathrm{n}}_{i}^{\mathrm{a}}-\mu^{\mathrm{b}} \hat{\mathrm{n}}_{i}^{\mathrm{b}}\right],
\end{gathered}
$$

where $U^{\mathrm{xy}}$ is the strength of interactions between a pair of particles with flavors $\mathrm{x}$ and $\mathrm{y}$ and $\mu^{\mathrm{x}}$ denotes the chemical potential of species $\mathrm{x}$. The Hamiltonian $\hat{\mathrm{H}}_{0}$ is easily diagonalized using the Fock state basis: $\hat{\mathrm{H}}_{0}\left|n^{\mathrm{a}}, n^{\mathrm{b}}\right\rangle=$ $E_{n^{\mathrm{a}}, n^{\mathrm{b}}}\left|n^{\mathrm{a}}, n^{\mathrm{b}}\right\rangle$. It is easy to see that $\hat{\mathrm{H}}_{0}$ allows for groundstate degeneracies which are lifted in the presence of a hopping term leading to different types of "magnetic" orderings in the Mott state 11].

As we wish to focus on the influence of gauge fields on the MI-SF transition, we shall first consider the parameter regime for which $\hat{\mathrm{H}}_{0}$ has a unique ground-state in the local limit. The simplest choice in this regard is to assume two independent, identical systems, i.e. $U^{\mathrm{aa}}=U^{\mathrm{bb}} \equiv U, \mu^{\mathrm{a}}=\mu^{\mathrm{b}} \equiv \mu$, and $U^{\mathrm{ab}}=0$. With this, $\hat{\mathrm{H}}_{0}$ describes a system which in its ground-state is in both flavors occupied by an equal number $n$ of particles, with $\mu / U<n<\mu / U+1$ as in the one-component BH model. This setup will be further investigated in Sections IIIB and IV, for the MI and SF phase, respectively.

In another scenario, investigated in Section IIIC and [V] we take into account repulsive interactions between the components $\left(U^{\mathrm{ab}}>0\right)$. In this case, a subspace spanned by all states $\left|n^{\mathrm{a}}, n^{\mathrm{b}}\right\rangle$ with $n^{\mathrm{a}}+n^{\mathrm{b}}=\tilde{n}$ forms the degenerate ground state manifold, where $\tilde{n}$ is the particle number per site. For $0<\mu / U<U^{a b} / U$, one single atom occupies each site, such that any linear combination $c_{1}|1,0\rangle+c_{2}|0,1\rangle$ at every site is a ground state of the unperturbed Hamiltonian $\hat{\mathrm{H}}_{0}$. The hopping lifts this degeneracy. As shown in Ref. 12, for small $\lambda \equiv U^{a b} / U$, an antiferromagnetic ordering is preferred, while for $\lambda \sim 1$, the system chooses a translational-invariant ferromagnetic phase. Between these two limits an XY ordering with $c_{1}=c_{2}=1 / \sqrt{2}$ occurs. In the rest of this work, we shall focus on the ferromagnetic and the XY phases, where the ground state preserves translational symmetry.

The kinetic part of the Hamiltonian is given by

$$
\hat{\mathrm{H}}_{1}=-\sum_{i, j}\left(\hat{\mathrm{a}}_{i}^{\dagger}, \hat{\mathrm{b}}_{i}^{\dagger}\right) J_{i j}\left(\begin{array}{c}
\hat{\mathrm{a}}_{j} \\
\hat{\mathrm{b}}_{j}
\end{array}\right) \text {, }
$$

where $J_{i j}=\delta_{<i j>} J \mathrm{e}^{-i\left(\boldsymbol{A}_{j} \cdot \boldsymbol{r}_{j}-\boldsymbol{A}_{i} \cdot \boldsymbol{r}_{i}\right)}$ is a nearest-neighbor hopping with a constant strength $J$, and we have chosen $\hbar=c=1$. The phase factor associated with the hopping is defined by the gauge potential $\boldsymbol{A}_{i}$, which we choose to be of the following form

$$
\boldsymbol{A}_{i}=\left(\alpha \sigma_{y}, \beta \sigma_{x}+2 \pi \boldsymbol{r}_{i} \cdot \hat{e}_{x} \Phi, 0\right),
$$

where $\sigma_{x, y}$ are the Pauli matrices, $\Phi$ is an Abelian flux, $\hat{e}_{x}$ denotes the unit vector along $x, \boldsymbol{r}_{i}$ is the spatial coordinate of site $i$, and $\alpha, \beta$ are parameters characterizing the non-Abelian vector potential. Although interesting anisotropy effects can be expected from choosing $\alpha \neq \beta$ [35, 36, in this work we shall consider $\alpha=\beta$ for simplicity. With this choice, the intra-species hopping terms $\left(\hat{\mathrm{a}}_{i}^{\dagger} \hat{\mathrm{a}}_{j}\right.$ and $\hat{\mathrm{b}}_{i}^{\dagger} \hat{\mathrm{b}}_{j}$ ) become proportional to $\cos \alpha$, while the inter-species hopping terms with non-Abelian vector potential $\left(\hat{a}_{i}^{\dagger} \hat{\mathrm{b}}_{j}\right.$ and $\left.\hat{\mathrm{b}}_{i}^{\dagger} \hat{\mathrm{a}}_{j}\right)$ become proportional to $\sin \alpha$. For $\alpha=0$ and $\Phi \neq 0$, we thus recover the Hofstadter problem of a constant magnetic field perpendicular to the twodimensional (2D) system in the Landau gauge [37. Note that also in the opposite limit, $\Phi=0$ and $\alpha \neq 0$, where the vector potential becomes constant, the non-Abelian character of the gauge potential, i.e. $\left[A_{i}, A_{j}\right] \neq 0$, yields a constant but finite gauge field. For the Abelian flux, we shall focus on $\Phi=p / q$, where $p$ and $q$ are co-prime integers. Most of our work has been done for $\Phi=0$ or $1 / 2$; however, the method developed here can be straightforwardly extended to other values of $\Phi$ as shown in Ref. 25 .

\section{MOTT INSULATING PHASE}

\section{A. Hopping expansion}

Our approach to study the full Hamiltonian $\hat{\mathrm{H}}=$ $\hat{\mathrm{H}}_{0}+\hat{\mathrm{H}}_{1}$ is based on a resummed hopping expansion as developed in Refs. 30 and 31 for the single species BH model. In this formalism, one considers the hopping term as a perturbation and focuses on the imaginarytime evolution of the operators: $\hat{a}(\tau)=\mathrm{e}^{\hat{\mathrm{H}}_{0} \tau} \hat{\mathrm{a}} \mathrm{e}^{-\hat{\mathrm{H}}_{0} \tau}$. 
Introducing artificial sources $j_{i}^{\mathrm{a}}(\tau), j_{i}^{\mathrm{b}}(\tau): \hat{\mathrm{H}}_{1}[\{j\}](\tau)=$ $\hat{\mathrm{H}}_{1}(\tau)+\sum_{i}\left(\bar{j}_{i}^{\mathrm{a}}(\tau) \hat{\mathrm{a}}_{i}(\tau)+\bar{j}_{i}^{\mathrm{b}}(\tau) \hat{\mathrm{b}}_{i}(\tau)+\right.$ h.c. $)$ with $\beta$ the inverse temperature, and $\{j\}$ denoting the set of all four currents, the free energy of the system can be written as a functional of the sources:

$$
\mathcal{F}[\{j\}]=-\frac{1}{\beta} \ln \operatorname{Tr}\left(\mathrm{e}^{-\beta \hat{\mathrm{H}}_{0}} \hat{\mathrm{T}}_{\tau} \mathrm{e}^{-\int_{0}^{\beta} \mathrm{d} \tau \hat{\mathrm{H}}_{1}[\{j\}](\tau)}\right),
$$

where $\hat{\mathrm{T}}_{\tau}$ indicates imaginary-time ordering. From this it can be directly seen that the derivatives $\beta \delta \mathcal{F} / \delta \bar{j}_{i}^{\mathrm{a}(\mathrm{b})}(\tau)=$ $\left\langle\hat{\mathrm{a}}_{i}\left(\hat{\mathrm{b}}_{i}\right)(\tau)\right\rangle=\Psi_{i}^{\mathrm{a}(\mathrm{b})}(\tau)$ yield the order parameter fields $\Psi_{i}^{\mathrm{a}(\mathrm{b})}(\tau)$ which vanish within the MI phase. For a description of the Mott physics, it is thus sufficient to expand $\mathcal{F}[\{j\}]$ up to second order in the currents. Furthermore, as quantum fluctuations of the hopping term scale down with dimension 38, in a two-dimensional system an expansion of $\mathcal{F}[\{j\}]$ up to first order in the hopping strength $J$ and a subsequent resummation is expected to yield qualitatively reasonable results. This is automatically achieved by performing a Legendre transformation iteratively in the hopping, which substitutes the source fields $\{j\}$ by the physical order-parameter field. Carrying out these steps, as detailed in Refs. 30 and 31, we finally obtain the effective action of the system up to second order in $J / U$ :

$$
S[\{\Psi\}]^{\mathrm{MI}}=\frac{1}{\beta} \sum_{i, j}\left(\bar{\Psi}_{i}^{\mathrm{a}}, \bar{\Psi}_{i}^{\mathrm{b}}\right)\left[\left(\hat{G}_{i j}^{0}\right)^{-1}-J_{i j}\right]\left(\begin{array}{c}
\Psi_{j}^{\mathrm{a}} \\
\Psi_{j}^{\mathrm{b}}
\end{array}\right)
$$

where $\hat{G}_{i j}^{0}=\left\langle\hat{\mathrm{T}}_{\tau} \hat{\mathrm{O}}_{i}^{\dagger}(\tau) \hat{\mathrm{O}}_{j}\left(\tau^{\prime}\right)\right\rangle_{0}$ is the unperturbed twopoint function. Here the operators $\hat{\mathrm{O}}_{i}^{\dagger}$ and $\hat{\mathrm{O}}_{j}$ may be of type $a$ or $b$, and the thermal average is with respect to $\hat{\mathrm{H}}_{0}$. Note that $\hat{G}^{0}$ is a function of $\tau-\tau^{\prime}$; thus $\left(\hat{G}^{0}\right)^{-1}$ is most easily found in frequency space. We point out that an alternative way to deriving the same effective action within a random phase approximation is described in Ref. 7 for the BH model, and can straightforwardly be generalized to systems with an Abelian gauge field [25]. From Eq. [5, we find that $\hat{G}=\left[\left(\hat{G}^{0}\right)^{-1}-J_{i j}\right]^{-1}$ is the resummed two-point function which can be used, for example, to find the boson momentum distribution [7, 25]

$$
n_{\boldsymbol{k}}=-\lim _{T \rightarrow 0} \frac{1}{\beta} \sum_{\omega_{\mathrm{M}}} \operatorname{Tr}\left[\hat{G}\left(\boldsymbol{k}, i \omega_{\mathrm{M}}\right)\right]
$$

These momentum distributions can be observed in timeof-flight (TOF) measurements, as we shall discuss later.

The excitation spectra of the bosons can be obtained from the poles of $\hat{G}$ or equivalently by setting up the equation of motion: $\delta S[\{\Psi\}]^{\mathrm{MI}} / \delta \bar{\Psi}^{\mathrm{a}, \mathrm{b}}=0$. In Fourier space, this reads

$$
\sum_{\boldsymbol{k}}\left[\delta_{\boldsymbol{k}, \boldsymbol{k}^{\prime}} \hat{G}^{0}\left(\omega_{\mathrm{M}}\right)^{-1}-J_{\boldsymbol{k}, \boldsymbol{k}^{\prime}}\right]\left(\begin{array}{c}
\Psi_{\boldsymbol{k}^{\prime}}^{\mathrm{a}}\left(\omega_{\mathrm{M}}\right) \\
\Psi_{\boldsymbol{k}^{\prime}}^{\mathrm{b}}\left(\omega_{\mathrm{M}}\right)
\end{array}\right)=0,
$$

where $J_{\boldsymbol{k}, \boldsymbol{k}^{\prime}}$ is the Fourier transform of $J_{i j}$. After an analytic continuation to real frequencies $i \omega_{\mathrm{M}} \rightarrow \omega+i \epsilon$, the solutions of Eq. (7) yield the dispersion relations. In the following subsections, we apply this general procedure to specific choices of gauge potentials and parameters $\lambda$ and $\mu$.

\section{B. Independent Species}

The simplest non-trivial parameter choice which we shall treat in this section corresponds to $\lambda=0$ and $0 \leq \mu / U \leq 1$. In this case, $\hat{\mathrm{H}}_{0}$ describes two independent standard $\mathrm{BH}$ systems with unique non-degenerate ground state having one boson of each species per site. The coupling between them is provided by the inter-species hopping terms arising from the non-Abelian gauge potential. For this case, it is clear that the unperturbed Green function has vanishing off-diagonal terms, i.e. $G_{12}^{0} \sim\left\langle\hat{\mathrm{a}}^{\dagger}(0) \hat{\mathrm{b}}(\tau)\right\rangle_{0}=0$ and $\hat{G}_{21}^{0} \sim\left\langle\hat{\mathrm{b}}^{\dagger}(0) \hat{\mathrm{a}}(\tau)\right\rangle_{0}=0$. From our symmetric choice of parameters, it also follows that $G_{11}^{0}=G_{22}^{0}$ rendering $\hat{G}^{0} \sim \mathbb{1}_{2 \times 2}$. Further, the site-factorizable nature of $\hat{H}_{0}$ guarantees that the diagonal elements of $\hat{G}^{0}$ are given by $\delta_{\boldsymbol{k}, \boldsymbol{k}^{\prime}}$ times a function $G^{0}\left(\omega_{\mathrm{M}}\right)$ of a single Matsubara frequency

$$
G^{0}\left(\omega_{\mathrm{M}}\right)=\sum_{n, m=0}^{\infty} \frac{\mathrm{e}^{\beta E_{n, m}}}{\mathcal{Z}^{0}}\left(\frac{n+1}{\Delta_{n+1}-i \omega_{\mathrm{M}}}-\frac{n}{\Delta_{n}-i \omega_{\mathrm{M}}}\right),
$$

where $\mathcal{Z}^{0}=\sum_{n, m=0}^{\infty} \mathrm{e}^{\beta E_{n, m}}$, and $\Delta_{n}=E_{n, m}-E_{n-1, m}$. In the zero-temperature limit, the Boltzmann sums in Eq. (8) reduce to a single term corresponding to the groundstate occupation numbers, $n=m=1$. In the following two subsections, we shall compute $\hat{G}$ for the two simplest choices of gauge potentials corresponding to a constant non-Abelian gauge field: the one without Abelian flux, $\Phi=0$, and that with an Abelian flux $\Phi=1 / 2$.

$$
\text { 1. } \Phi=0
$$

For a constant gauge potential without Abelian flux, $\Phi=0$, the hopping matrix is diagonal in momentum space, i.e.,

$$
\begin{aligned}
J_{\boldsymbol{k}, \boldsymbol{k}^{\prime}}= & 2 J\left\{\cos \alpha\left[\cos \left(k_{x}\right)+\cos \left(k_{y}\right)\right] \mathbb{1}\right. \\
& \left.-\sin \alpha\left[\sin \left(k_{x}\right) \sigma_{y}+\sin \left(k_{y}\right) \sigma_{x}\right]\right\} \delta_{\boldsymbol{k}, \boldsymbol{k}^{\prime}},
\end{aligned}
$$

where here and in the rest of the paper, we have set the lattice spacing $a \equiv 1$. As the unperturbed Green function $\hat{G}^{0}$ is already diagonal, we need to diagonalize only the hopping matrix. This yields

$$
\begin{aligned}
E_{\boldsymbol{k}}^{ \pm}= & 2 J\left[\cos (\alpha)\left[\cos \left(k_{x}\right)+\cos \left(k_{y}\right)\right]\right. \\
& \left. \pm \sin (\alpha) \sqrt{\cos ^{2}\left(k_{x}\right)+\cos ^{2}\left(k_{y}\right)}\right]
\end{aligned}
$$




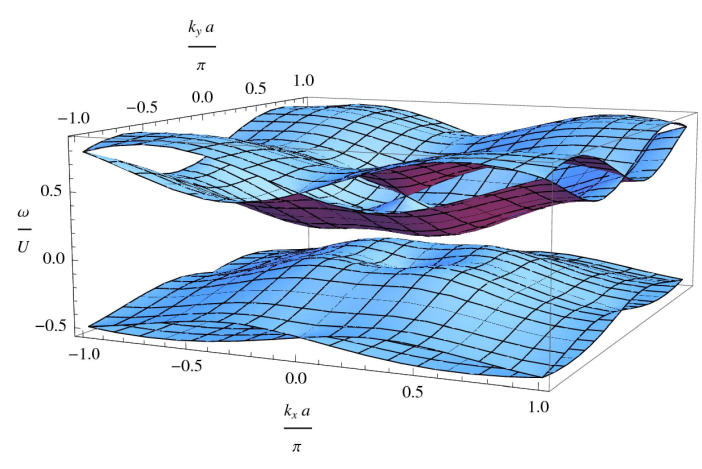

FIG. 1. Boson energy dispersion for $\Phi=0, \alpha=1, \mu / U=$ 0.41 , and $J / U=0.05$. See text for details.

The energy bands of the system at zero temperature are thus given by $\left.\left[G^{0}(\omega+i \eta)\right]^{-1}\right|_{T=0}-E_{k}^{ \pm}=0$, where we have Wick rotated back to real frequency. This yields two quadratic equations

$$
\begin{aligned}
& \omega^{2}+\omega\left(2 \mu-U+E_{\boldsymbol{k}}^{+}\right)+\mu^{2}-\mu U+(\mu+U) E_{\boldsymbol{k}}^{+}=0 \\
& \omega^{2}+\omega\left(2 \mu-U+E_{\boldsymbol{k}}^{-}\right)+\mu^{2}-\mu U+(\mu+U) E_{\boldsymbol{k}}^{-}=0
\end{aligned}
$$

leading to four energy bands shown in Fig. 1. Two of these bands occur at $\omega>0$ and the other two at $\omega<0$, so that they may be interpreted as particle/hole excitation spectra of the system. We note that as $\alpha \rightarrow 0$, the particle and the hole spectra become increasingly similar and ultimately indistinguishable for $\alpha=0$ yielding the standard dispersion of MI bosons with no gauge potential 7 . Furthermore, the particle and hole excitations have a gap in the Mott phase which closes as one approaches the superfluid-insulator transition by increasing $J / U$. Beyond the transition point, which occurs at $J=J_{c}$, the solutions of Eq. (11) are complex, indicating instability of the Mott phase.

The superfluid-insulator phase boundary, as obtained by the procedure described above, is shown in Fig. 2. We find that the phase boundary has the usual lobe structure. However, the value of $J_{c}$ at the tip of the Mott lobe is strongly influenced by $\alpha$ and can thus be tuned by varying the strength of the gauge field. This leads to re-entrant superfluid-insulator transitions in the system by variation of $\alpha$, provided that $J$ is appropriately fixed at (say) $J=J_{c}(\alpha=0.2 \pi)$ as can be seen from Fig. 2. We also note that the universality class of the superfluid-insulator transition has the same properties as in the standard $\mathrm{BH}$ case [4. At the lobe tip, the additional particle-hole symmetry renders the dynamical critical exponent $z$ of the transition to be unity; at other points, $z=2$.

One of the key difference of the superfluid-insulator transition in the present system from the normal $\mathrm{BH}$ model is that the position of the minima of the low-energy excitations at and near the critical point in the first Brillouin zone are at finite momenta and strongly depend on
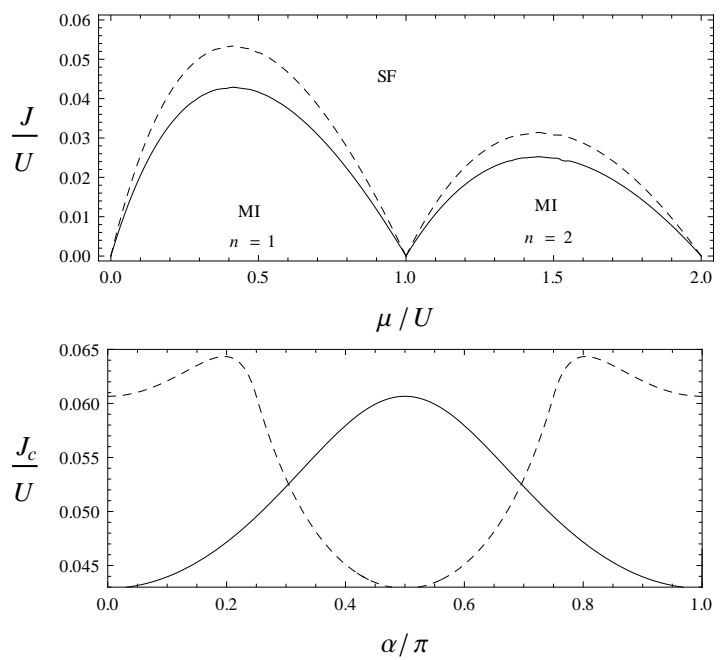

FIG. 2. Up The Mott lobes with $\alpha$ dependent heights. The solid (dashed) lines indicate the lobes for $\alpha=0(1)$. Down: Value of $J_{c}$ at the tip of the $n=1$ Mott lobe as a function of $\alpha$. The solid (dashed) lines correspond to $\Phi=0(1 / 2)$.

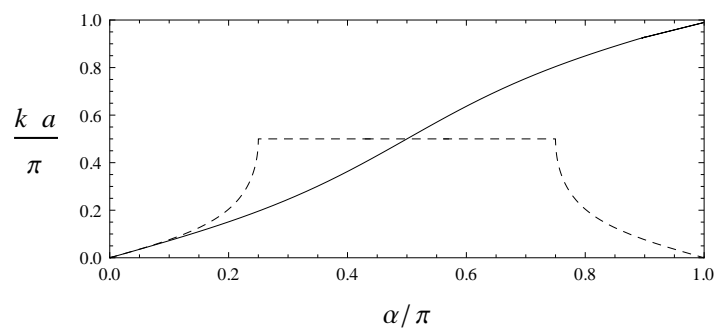

FIG. 3. The position of the boson energy minima at finite wavevector parametrized by $k$ (see text) in systems without flux (solid line) and with $1 / 2$ flux (dashed line). In the latter, a QPT of the excited band occurs at $\alpha=\pi / 4$.

$\alpha$ (see Fig. 3). Since the position of these minima correspond to precursor peaks of the bosons near the critical point [7, this feature is reflected in the momentum distribution of the bosons in the Mott phase near the transition. From the Green function $G$, we compute the momentum distribution using Eq. (6). The results of this calculation are shown in the top panel of Fig. 4. We find that at finite $\alpha$, the precursor peaks of the momentum distribution at $J \approx 0.97 J_{c}(\alpha)$ are at finite momenta, reflecting the fact that the subsequent condensation of the bosons at $J=J_{c}(\alpha)$ will occur at non-zero momenta. We note that such a pattern in the momentum distribution should be easily picked up in TOF experiments. Since standard TOF experiments will measure the distribution of both species simultaneously, the presence of the noncondensing mode will slightly reduce the visibility of the pattern. However, we expect that one should be able to easily subtract this background in order to observe the sharp peaks of the condensing mode. The most significant feature distinguishing the non-Abelian scenario from the known standard one, is the number of peaks which 
in general is quadrupled by the non-Abelian gauge field. We predict this feature to be clearly observable in TOF experiments.

$$
\text { 2. } \Phi=1 / 2
$$

Turning to the case with an Abelian flux $\Phi$ in the gauge potential of Eq. (3), we find the general structure for the hopping matrix to be given by:

$$
J_{\boldsymbol{k}, \boldsymbol{k}^{\prime}}=A \delta_{\boldsymbol{k}, \boldsymbol{k}^{\prime}}+B \delta_{k_{y}, k_{y}^{\prime}} \delta_{k_{x}, k_{x}^{\prime}+2 \pi \Phi}+C \delta_{k_{y}, k_{y}^{\prime}} \delta_{k_{x}, k_{x}^{\prime}-2 \pi \Phi},
$$

making Eq. (7) to have off-diagonal terms connecting momenta in the magnetic Brillouin zone which differ by $\pm 2 \pi \Phi$. Here $A, B$ and $C$ are functions of momentum which will be specified later. For a generic $\Phi=p / q$, the periodicity of the lattice $\Psi_{\boldsymbol{k}}=\Psi_{\boldsymbol{k}+2 \pi n \boldsymbol{e}_{x}}$ ensures that Eq. (7) leads to a set of $q$ closed equations. To write them down, we introduce the notation $\boldsymbol{\Psi}_{\boldsymbol{k}+n \times 2 \pi \Phi \boldsymbol{e}_{x}}=\boldsymbol{\Psi}_{\boldsymbol{k}, n}$ with $n=0, \cdots, q-1$. In this notation, the equation of motion can be written in the Harper-like form:

$$
M\left(k_{x}, n\right) \boldsymbol{\Psi}_{\boldsymbol{k}, n}+\mathrm{e}^{i a k_{y}} N \boldsymbol{\Psi}_{\boldsymbol{k}, n-1}+\mathrm{e}^{-i a k_{y}} N^{*} \boldsymbol{\Psi}_{\boldsymbol{k}, n+1}=0,
$$

with

$$
\begin{aligned}
& M\left(k_{x}, n\right) \equiv\left[G^{0}\left(\omega_{\mathrm{M}}\right)\right]^{-1} \mathbb{1}_{2 \times 2}-2 J \\
& \times\left(\begin{array}{cc}
\cos \left(a k_{x}+2 \pi \Phi n\right) \cos \alpha & i \sin \left(a k_{x}+2 \pi \Phi n\right) \sin \alpha \\
-i \sin \left(a k_{x}+2 \pi \Phi n\right) \sin \alpha & \cos \left(a k_{x}+2 \pi \Phi n\right) \cos \alpha
\end{array}\right),
\end{aligned}
$$

and

$$
N \equiv-J\left(\begin{array}{cc}
\cos \beta & i \sin \beta \\
i \sin \beta & \cos \beta
\end{array}\right)
$$

As each equation in Eq. 13 is a two-component equation, for a flux of $\Phi=p / q$, we have $2 q$ closed equations. Here we shall focus on $p=1, q=2$ which allows us to find the solutions of these equations analytically via diagonalization of a $4 \times 4$ hopping matrix.

We find that the presence of the magnetic flux $\Phi=1 / 2$ splits each band, so that we now have four particle and four hole excitations. Again the most relevant bands are the particle (hole) excitation at lowest (highest) frequency. The gap between these excitations closes at $J=J_{c}$ leading to a second-order QPT separating the MI and the SF phases. The lobe structure of the phase boundary (and also the universality class of the transition) remain unchanged by the flux, as can be seen from Fig. 2. We note however that the plot of $J_{c}(\alpha)$ as a function of $\alpha$, shown in the bottom panel of Fig. 2, has a qualitatively different behavior compared to the case without Abelian flux discussed previously; nonetheless, the system will show similar re-entrant superfluid-insulator transitions as $\alpha$ is varied for a fixed $J$.

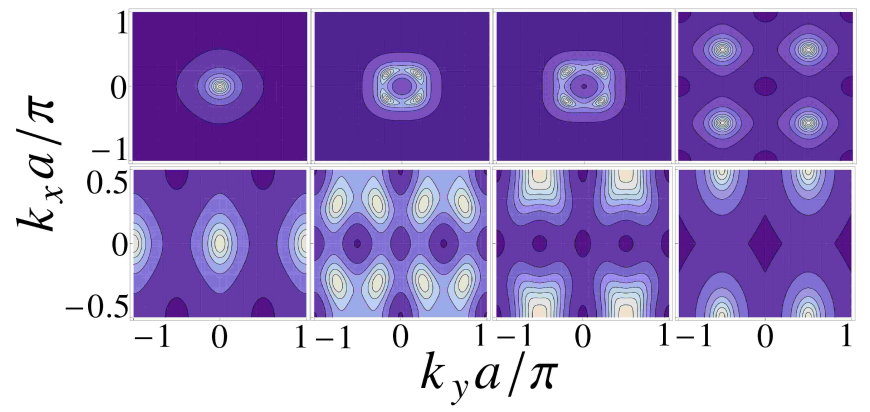

FIG. 4. Momentum distributions of the bosons at $J \approx$ $0.97 J_{c}$. Bright regions correspond to high densities. The upper (lower) rows correspond to $\Phi=0(1 / 2)$. The nonAbelian field strengths $\alpha$ are, from left to right in each panel, $0,0.7,0.8$, and $\pi / 2$.

The most interesting difference between $\Phi=0$ and $\Phi=1 / 2$ concerns the number and positions of the extrema of the particle and hole excitations. We find that within the first magnetic Brillouin zone $\left(k_{x} \in\right.$ $\left.[-\pi / q, \pi / q], \quad k_{y} \in[-\pi, \pi]\right)$ and in the absence of a non-Abelian field $(\alpha=0)$, there are two extrema at $\boldsymbol{k}=(0,0),(0, \pm \pi)$, in agreement with Ref. 25. Denoting these three points in the Brillouin zone as $\boldsymbol{k}_{0}, \boldsymbol{k}_{+}$, and $\boldsymbol{k}_{-}$, the extrema for non-zero $\alpha$ can be shown to occur at $\boldsymbol{k}_{0}+( \pm k, \pm k), \boldsymbol{k}_{+}+( \pm k,-k)$, and $\boldsymbol{k}_{-}-( \pm k,+k)$, where $k$ as a function of $\alpha$ is plotted in Fig. 3 . From this plot, we find that as long as $\alpha<\pi / 4$, we have eight extrema. When $\alpha=\pi / 4$, we get $k=\pi / 2$ and the extrema are completely shifted to the zone edges, i.e. again we have only two extrema per Brillouin zone. For $\pi / 4<\alpha<3 \pi / 4$, a plateau with a single fixed extremum is found. The derivative $\mathrm{d} k / \mathrm{d} \alpha$ diverges at $\alpha \rightarrow \pi / 4$ which means that at this critical value of the non-Abelian field the global minima of the excited bands abruptly change their position. Thus a slight change in the gauge field strength is expected to completely modify the dynamical behavior of the system. This remarkable behavior can be directly observed in the momentum distribution shown in the bottom panel of Fig. 4. In particular, the abrupt change in the pattern of the momentum distribution when the flux is varied across $\pi / 4$ reflects the sudden change in the position of the band minima with small change in $\alpha$. This behavior is reminiscent of the QPT of excited states discussed in Ref. 32. Also the abrupt sign reversal of the Hall conductivity at half filling in a system of hard-core bosons subjected to a gauge field as studied in Ref. 33 falls into this category of phenomena where some control parameter modifies the system's dynamics in a discontinuous way. 


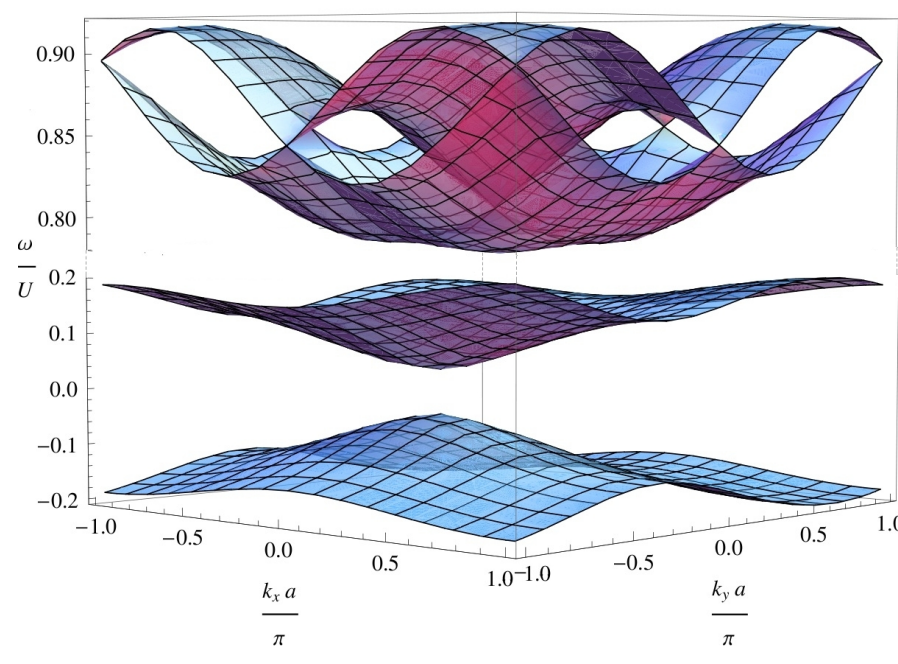

FIG. 5. The excitation spectrum of the bosons in the XY phase system for $\alpha=1, \Phi=0, \mu / U=0.15, \lambda=0.3$, and $J / U=0.021$.

\section{XY configuration}

In this section, we consider the case where $\lambda>0$ such that the ground state of the two-species model without the gauge field correspond to the XY phase discussed in Ref. 12, where the bosons are in the superposition of both the states. Consequently, $\hat{G}^{0}$ will have non-zero off-diagonal components and one needs to diagonalize the full matrix $\left(\hat{G}^{0}\right)^{-1}-J_{\boldsymbol{k}}$ in the presence of the non-Abelian flux.

We begin by computing the elements of $\hat{G}^{0}$ at $T=0$ which are given by

$$
\hat{G}^{0}=\left(\begin{array}{ll}
\left\langle\hat{\mathrm{T}}_{\tau} \hat{\mathrm{a}}_{i}^{\dagger}(\tau) \hat{\mathrm{a}}_{i}\left(\tau^{\prime}\right)\right\rangle_{\mathrm{GS}} & \left\langle\hat{\mathrm{T}}_{\tau} \hat{\mathrm{a}}_{i}^{\dagger}(\tau) \hat{\mathrm{b}}_{i}\left(\tau^{\prime}\right)\right\rangle_{\mathrm{GS}} \\
\left\langle\hat{\mathrm{T}}_{\tau} \hat{\mathrm{b}}_{i}^{\dagger}(\tau) \hat{\mathrm{a}}_{i}\left(\tau^{\prime}\right)\right\rangle_{\mathrm{GS}} & \left\langle\hat{\mathrm{T}}_{\tau} \hat{\mathrm{b}}_{i}^{\dagger}(\tau) \hat{\mathrm{b}}_{i}\left(\tau^{\prime}\right)\right\rangle_{\mathrm{GS}}
\end{array}\right)
$$

For the XY ground state $|\mathrm{GS}\rangle=\frac{1}{\sqrt{2}}(|10\rangle+|01\rangle)$, discussed in Ref.12, this matrix reads

$$
\begin{aligned}
\hat{G}^{0}(i \omega)= & {\left[\frac{1}{2 U \lambda-2(\mu+i \omega)}+\frac{1}{U-\mu-i \omega}+\frac{1}{2 \mu+2 i \omega}\right] \mathbb{1} } \\
& +\left[-\frac{U \lambda}{2(\mu+i \omega)(-U \lambda+\mu+i \omega)}\right] \sigma_{x}
\end{aligned}
$$

where $\mathbb{1}$ is the unit matrix and $\sigma_{x}$ denotes the Pauli matrix. Inserting Eq. (17) into Eq. (7), and considering the case $\Phi=0$, we find that the presence of the off-diagonal elements in $\hat{G}^{0}$ leads to two independent equations for the band dispersions given by

$$
\begin{aligned}
& M_{1}+A_{k}+\sqrt{M_{2}^{2}+\left|B_{k}\right|^{2}+M_{2}\left(B_{k}+B_{k}^{*}\right)}=0 \\
& M_{1}+A_{k}-\sqrt{M_{2}^{2}+\left|B_{k}\right|^{2}+M_{2}\left(B_{k}+B_{k}^{*}\right)}=0
\end{aligned}
$$
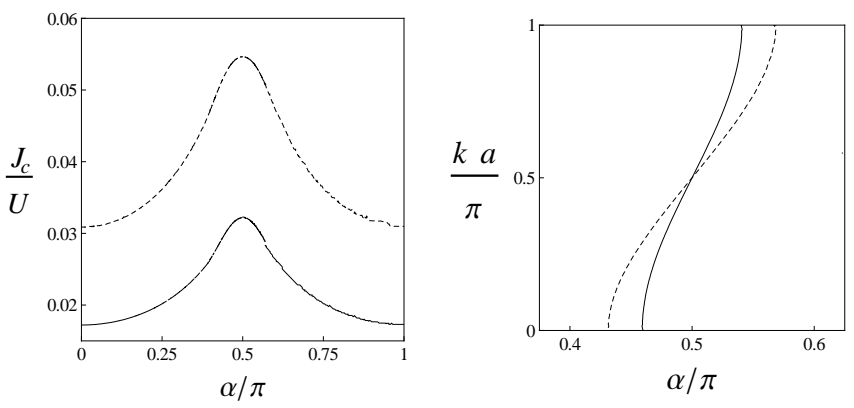

FIG. 6. Left: The critical hopping $J_{c}$ marking the tip of the lobe for $\lambda=0.3$ (solid line) and $\lambda=0.6$ (dashed line) as a function of $\alpha$. Right: The minima (maxima) of the lowest (highest) particle (hole) excitation are found at $k_{y} a=-\alpha$ and $k= \pm k_{x}$ as shown in the plot (the solid line corresponds to $\lambda=0.3$, the dashed line to $\lambda=0.6)$. It is different from 0 or $\pm \pi$ only within a small region around $\alpha=\pi / 2$, in which the single extremum suddenly splits into two.

where

$$
\begin{aligned}
M_{1} & =\frac{(\omega+\mu-U)\left(U^{2} \lambda+U \lambda(\omega+\mu)-2(\mu+\omega)^{2}\right)}{2 U^{2} \lambda-2(\mu+\omega)^{2}} \\
M_{2} & =\frac{U \lambda(\mu+\omega-U)^{2}}{2 U^{2} \lambda-2(\mu+\omega)^{2}} \\
A_{k} & =2 J \cos \alpha\left(\cos k_{x}+\cos k_{y}\right) \\
B_{k} & =2 J \sin \alpha\left(\sin k_{y}+i \sin k_{x}\right)
\end{aligned}
$$

Solving the first of these equations, we find two positive $(E>0)$ and one negative $(E<0)$ solutions, while the second equation has solely one positive solution which is the second highest band. The resulting bandstructure is shown in Fig. 5. Note that here the presence of the off-diagonal component of the Green function which originates from the XY ground state leads to more particle-like than hole-like excitations. This feature is a consequence of particle-hole asymmetry originating from $U^{\mathrm{ab}} \neq U$. Also, the splitting of the two highest particlelike excitations is a consequence of the non-Abelian nature of the hopping. This splitting vanishes in the limit $\alpha \rightarrow 0$.

For the QPT into the SF state these higher modes again do not play a role. The Mott lobe, on which at least one mode becomes gapless, now extends from $0<$ $\mu<\lambda U$. The value of $J_{c}$ marking the height of the lobe depends on both $\alpha$ and $\lambda$, as illustrated on the left panel of Fig. 6. Furthermore, as in the cases studied before, we find that the minima of the dispersion occur at finite wavevectors. However, in contrast to the cases studied before, the band spectrum in the XY phase is not symmetric under $k_{y} \rightarrow-k_{y}$. This property of the dispersion can be traced back to $G$ since $B_{k}$ in Eq. (19) is not invariant under such a transformation.

The above-mentioned properties of the dispersion of the particle- and hole-like excitations are reflected in the nature of the momentum distribution of the bosons in the Mott phase near the quantum critical point. We find 


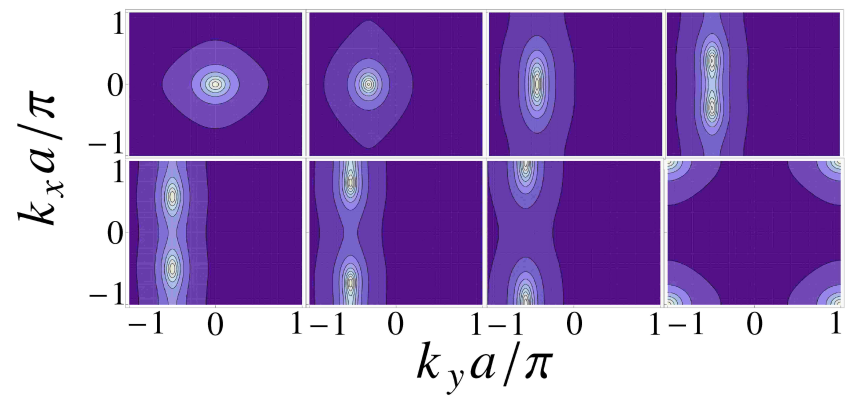

FIG. 7. Momentum distributions at $J \approx 0.97 J_{c}$ in $\mathrm{XY}$ system for $\lambda=0.3$. The bright regions correspond to high densities. The field strengths $\alpha$ are, from left to right, $0,1,1.4,1.5, \pi / 2,1.65,1.75$, and $\pi$.

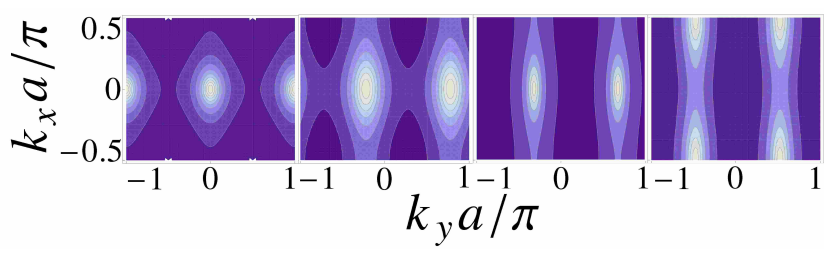

FIG. 8. The momentum distributions of the bosons at $J \approx$ $0.97 J_{c}$ in the XY system with $\Phi=1 / 2$ for $\lambda=0.3$ and field strengths $\alpha$, from left to right, $0,0.7,1$, and 1.5 .

that for any given $\alpha$, the condensing modes are located at $k_{y}=-\alpha$. As shown in Fig. 7, there are no analogous peaks at $k_{y}=\alpha$ which clearly reflects the breaking of the parity symmetry discussed above. Also we note that for the range $0 \leq \alpha \lesssim 1.5(1.65 \lesssim \alpha \leq \pi)$, there is a single condensing mode at $k_{x}=0\left(k_{x}= \pm \pi\right)$. Around $\alpha=\pi / 2$, however, the condensing mode splits into two at momenta $\boldsymbol{k}=( \pm k,-\alpha)$, where $k$ is plotted on the right panel of Fig. 6 as a function of $\alpha$. We note that such a splitting can modify the dynamical behavior of the system.

Finally, we study the influence of a magnetic flux on the XY system, as done before for $\lambda=0$. As expected we find the Abelian flux $\Phi=1 / 2$ to split each band into two, such that the system exhibits two hole and six particle excitations. As before, for $\alpha=0$, the presence of this flux provides the band structure with two extrema at $\boldsymbol{k}=(0,0)$ and $(0, \pm \pi)$. The resulting momentum distribution near condensation is shown in Fig. 8. As $\alpha$ is increased, the position of the peaks of the momentum distribution initially shifts along $k_{y}$. However, close to $\alpha=\pi / 2$, the peaks split along $k_{x}$ leading to four peaks which finally reach the zone edge $\boldsymbol{k}=( \pm \pi / 2, \pm \pi / 2)$ at $\alpha=\pi / 2$. These features, as in the case of $\Phi=0$, should be experimentally observable via standard TOF experiments.

\section{SUPERFLUID PHASE}

In this section, we investigate the nature of the SF phase into which the transition takes place. We note that, as pointed out in Ref. 7 and 25, the quadratic action (Eq. (5)) does not capture the physics of the ordered phase but needs to be supplemented by the quartic term at the mean-field level. These terms can be directly computed using the methods of Ref. 7 and 25] within a strong coupling expansion; however, it is often enough to guess their forms from the symmetry of the underlying Hamiltonian. In this section, we are going to take the latter route and chart out the characteristics of the resulting SF phase.

For determining the order parameters in the SF phase we need to construct the quartic part of the effective action in terms of the order parameter fields and minimize it. To this end, we first rewrite the quadratic action by diagonalizing its kernel as shown in Section III] Let us denote the eigenvalues and eigenmodes of the quadratic action as $m_{n, \boldsymbol{k}}(\omega)$ and $\Psi_{n, \boldsymbol{k}}(\omega)$, respectively. In the case when the Abelian flux is $\Phi=p / q, n$ varies from 0 to $2 q-1$. Note that here we have adopted the convention that for $\Phi=0$, the system remains with only two eigenmodes, so that we have $n=0,1$. In the zero-temperature limit we may then write,

$$
S^{\mathrm{MI}}=\sum_{n=0}^{2 q-1} \sum_{\boldsymbol{k}} \int \mathrm{d} \omega m_{n, \boldsymbol{k}}(\omega)\left|\Psi_{n, \boldsymbol{k}}(\omega)\right|^{2} .
$$

Here, the sum over $\boldsymbol{k}$ is restricted to the first magnetic Brillouin zone. The fourth-order term can be written in this basis as

$$
S^{(4)}=g / 2 \sum_{n=0}^{2 q-1} \sum_{i} \int_{0}^{\beta} \mathrm{d} \tau\left|\bar{\Psi}_{n}\left(\boldsymbol{r}_{i}, \tau\right) \cdot \Psi_{n}\left(\boldsymbol{r}_{i}, \tau\right)\right|^{2},(21)
$$

where we have transformed the $\Psi_{n}$ and $\bar{\Psi}_{n}$ fields to real space. Here, $g>0$ is the exact two-particle vertex function of the bosons in the local limit, which has been computed in Refs. [7, 31] and $i$ denotes lattice sites.

With this the SF action may be written as

$$
S^{\mathrm{SF}}=S^{\mathrm{MI}}+S^{(4)} .
$$

Now we note that at the onset of superfluidity only one of the eigenmodes condenses; therefore, it is possible to analyze the SF phase within a mean-field approximation by ignoring the other modes. This observation allows us to get rid of the $n$-sum over all bands in Eq. 21]:

$$
S^{(4)}=g / 2 \sum_{i} \int_{0}^{\beta} \mathrm{d} \tau\left|\bar{\Psi}\left(\boldsymbol{r}_{i}, \tau\right) \cdot \Psi\left(\boldsymbol{r}_{i}, \tau\right)\right|^{2} .
$$

In the presence of a non-Abelian gauge field with magnetic flux $\Phi=0$, or flux $\Phi=p / q=1 / 2$, we have one or more than one minima of the boson energy spectrum depending upon the non-Abelian field strength $\alpha$. If the particle/hole modes consist of $s$ degenerate minima, then the corresponding Ginzburg-Landau theory can be expressed by $s$ low-energy fluctuating two-component fields (order parameters) $\phi_{n}(r, t)$ around these minima [25, 26]:

$$
\Psi\left(\boldsymbol{r}_{i}, t\right)=\sum_{n=1}^{s} \chi_{n}\left(\boldsymbol{r}_{i}\right), \phi_{n}\left(\boldsymbol{r}_{i}, t\right),
$$


where we have Wick rotated to real time. The coefficients $\chi\left(\boldsymbol{r}_{i}\right)$ are the real space eigenfunctions corresponding to the minimum energy band at $\boldsymbol{k}=\left(k_{x}^{\min }, k_{y}^{\min }\right)$ which can be expressed as:

$$
\chi_{n}\left(\boldsymbol{r}_{i}\right)=\sum_{l=0}^{q-1} c_{l} \mathrm{e}^{\left(i k_{x}^{\min }+2 \pi l / q\right) x_{i}} \mathrm{e}^{i k_{y}^{\min } y_{i}},
$$

Note that the sum in the above expression is restricted to $q$ terms, since the functions $\chi_{n}\left(\boldsymbol{r}_{i}\right)$ describe only the part of the spatial dependence of $\Psi\left(\boldsymbol{r}_{i}, t\right)$ that can be factored out for each term in the sum in Eq. (24). Here $c_{l}$ denotes the components of eigenvectors corresponding to the minimum energy band at $\boldsymbol{k}=\left(k_{x}^{\min }, k_{y}^{\min }\right)$.

In general, the quartic part of the Landau-Ginzburg action is difficult to obtain, since it is restricted only by the invariance under projective symmetry group (PSG) of the underlying square lattice [39. The elements of PSG include in this case translations along the $x$ and $y$ axes, rotation by $\pi / 2$ around the $z$ axis, and reflections about $x$ and $y$ axes. In our case, the situation is, however, much simpler, since we know the microscopic form of the quartic action, Eq. (21). We may therefore substitute Eq. (24) into Eq. (21), and obtain the explicit form of the quartic action in terms of the order parameters $\phi_{n}\left(\boldsymbol{r}_{i}, t\right)$. We can then find the saddle point of the total action with respect to $\phi_{n}\left(\boldsymbol{r}_{i}, t\right)$, and thus directly calculate the order parameters in the SF phase.

Let us first consider the case where the number of both flavors at each site is equal, as discussed in Sec. IIIB. If the boson spectrum has one minimum in the magnetic Brillouin zone, then the corresponding low-energy field can be written as

$$
\psi\left(\boldsymbol{r}_{i}, t\right)=\chi_{1}\left(\boldsymbol{r}_{i}\right) \phi_{1}\left(\boldsymbol{r}_{i}, t\right),
$$

and thus the SF density reads

$$
\rho_{s}=|\langle\psi\rangle|^{2}=\left|\sum_{l=0}^{q-1} c_{l} \mathrm{e}^{\left(i k_{x}^{\min }+2 \pi l / q\right) x_{i}} \mathrm{e}^{i k_{y}^{\min } y_{i}}\right|^{2}\left|\phi_{1}\right|^{2} .
$$

For $\Phi=0$, we can get rid of the sum in Eq. (25) and $\rho_{s}$ is simply equal to $\left|\phi_{1}\right|^{2}$, which has no modulation along $x$. In contrast, for $\Phi=p / q=1 / 2$, we find that $\rho_{s}$ displays a spatial pattern.

Next, we consider the case where there are two minima at $\boldsymbol{k}_{1}^{\min }=(\pi / 2, \pi / 2)$ and $\boldsymbol{k}_{2}^{\min }=(\pi / 2,-\pi / 2)$ within the magnetic Brillouin zone for $\alpha=\pi / 4$ and $\Phi=1 / 2$. Note that these are on the zone edge, such that the minima at the opposite edge are equivalent. In this case, $\psi\left(\boldsymbol{r}_{i}, t\right)=\chi_{1}\left(\boldsymbol{r}_{i}\right) \phi_{1}\left(\boldsymbol{r}_{i}, t\right)+\chi_{2}\left(\boldsymbol{r}_{i}\right) \phi_{2}\left(\boldsymbol{r}_{i}, t\right)$. Following the coarse-graining procedure charted out in Ref. 25, we find that the SF ground state corresponds to the condensation of any one of the low-energy fluctuating fields $\left\langle\phi_{1}\right\rangle \neq 0,\left\langle\phi_{2}\right\rangle=0$ or $\left\langle\phi_{1}\right\rangle=0,\left\langle\phi_{2}\right\rangle \neq 0$. The corresponding plot for $\alpha=\pi / 4$ and $\Phi=1 / 2$ in Fig. 9 a, shows a similar periodic pattern as found for a single minimum for $\Phi=1 / 2$. Similar analysis can be done for four minima at $( \pm \pi / 2, \pm \pi / 2)$ for $\alpha=\pi / 2$ and $\Phi=0$; in this case
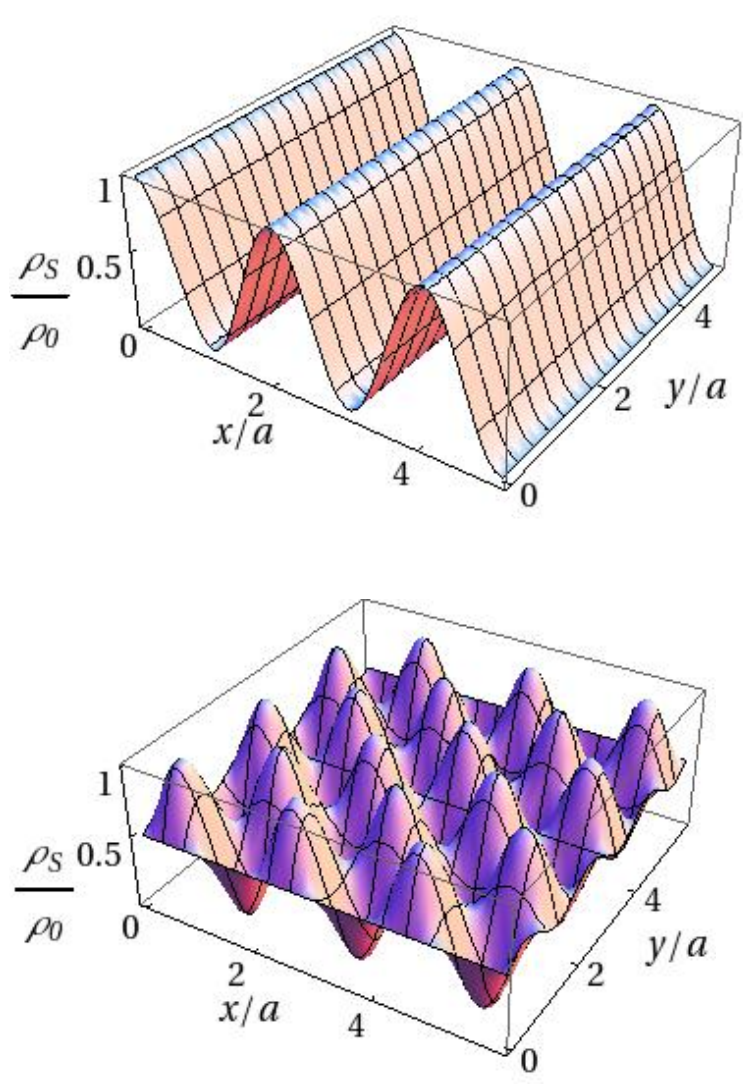

FIG. 9. a) Plot of SF density $\rho_{s} / \rho_{0}$ for $\Phi=1 / 2$ and $\alpha=\pi / 4$ at $\boldsymbol{k}^{\min } \equiv(\pi / 2, \pm \pi / 2)$ for even filling. b) Same plot for $\Phi=$ $1 / 2$ and $\alpha=\pi / 2$ at $\boldsymbol{k}^{\mathrm{min}} \equiv(\pi / 2, \pm \pi / 2)$ for XY phase.

we find that only one out the four field condenses; consequently there is no modulation of SF density. Note that we have restricted ourselves so far to the minima occurring at the wavevectors $\left(\pi / s_{1}, \pi / s_{2}\right)$, where $s_{1,2}= \pm 1$. In principle the analysis can be extended to the situations when the minima occur at $(\gamma \pi / 2, \delta \pi / 2)$ with rational and small $\gamma$ and $\delta$; however, the analysis becomes technically involved and we have not attempted that in this work. We do not have general understanding of implementing the above-mentioned coarse-graining procedure for irrational $\gamma, \delta$.

Finally, we briefly comment on the SF density in the $\mathrm{XY}$ phase. Following the procedure discussed before, we again find a constant SF density for any non-Abelian gauge field with $\Phi=0$. For $\alpha=\pi / 2$ and $\Phi=1 / 2$, there are two minima of the spectrum, and we find that the SF ground state corresponds to the condensation of both fields around these minima. The corresponding plot for $\alpha=\pi / 2$ is shown in Fig. $9 \mathrm{~b}$.

Thus we generically find that in the presence of a nonAbelian gauge field, the SF density displays a spatial periodic pattern if there is a finite flux $(\Phi=1 / 2)$; however, there is no such modulation without flux, $\Phi=0$. The method that has been discussed here can be used for any filling fraction $\Phi=p / q$. We expect different spatial patterns of the SF density for other $\Phi$, and leave the detailed analysis of it for future study. 


\section{CONCLUSION}

In conclusion, we have studied the Mott phases and the superfluid-insulator transition of strongly-interacting two-species bosons in the presence of a non-Abelian gauge field. We have shown that such a system of bosons is expected to display novel patterns of precursor peaks in the momentum distribution in the Mott phase close to the superfluid-insulator critical point. We have also demonstrated the presence of a re-entrant superfluidinsulator transition as the strength of the non-Abelian field is varied for a fixed hopping amplitude. Finally, we have found that the presence of an additional Abelian field with $\Phi=1 / 2$, leads to spatial modulation of the superfluid density in the SF phase near the critical point; however, no such modulation is expected for $\Phi=0$. We note that the precursor peaks in the momentum dis- tribution and the presence of the re-entrant superfluidinsulator transition can be easily detected in standard TOF experiments. Finally, we point out that we have restricted ourselves to model parameters for which the Mott phase has translational symmetry; it will be interesting to extend our study to the case where the Mott state has broken translational symmetry. We leave this as a subject of future study.

\section{ACKNOWLEDGEMENTS}

We acknowledge financial support by ERC Advanced Grant QUAGUATUA, EU Grant STREP NAMEQUAM, EU IP AQUTE, Spanish MINCIN Grant FIS2008-00784 (TOQATA), Consolider-Ingenio 2010 QOIT, the Alexander von Humboldt Foundation. KS thanks DST, India for support under grant SR/S2/CMP001/2009.
[1] M. Greiner, O. Mandel, T. Esslinger, T. W. Hänsch, and I. Bloch, Nature 415, 39 (2002).

[2] S. Sachdev, Quantum Phase Transitions (Cambridge University Press, 1999).

[3] M. Lewenstein, A. Sanpera, V. Ahufinger, B. Damski, A. Sen(De), and U. Sen, Adv. Phys. 56, 243 (2007).

[4] M. P. A. Fisher, P. B. Weichman, G. Grinstein, and D. S. Fisher, Phys. Rev. B 40, 546 (1989).

[5] R. Pandit, K. Seshadri, H. R. Krishnamurthy, and T. V. Ramakrishnan, Europhys. Lett. 22, 257 (1993).

[6] D. Jaksch, C. Bruder, J. I. Cirac, C. W. Gardiner, and P. Zoller, Phys. Rev. Lett. 81, 3108 (1998).

[7] K. Sengupta and N. Dupuis, Phys. Rev. A 71, 033629 (2005).

[8] C. Trefzger and K. Sengupta, Phys. Rev. Lett. 106, 2095702 (2011).

[9] Y. Kato, N. Kawashima, J. Freericks, H. R. Krishnamurthy, and N. Trivedi, Phys. Rev. A 79, 053631 (2009).

[10] W. D. Phillips, I. B. Spielman, and J. V. Porto, Phys. Rev. Lett. 98, 080404 (2007).

[11] E. Altman, W. Hofstetter, E. Demler, and M. D. Lukin, New Journal of Physics 5, 113 (2003).

[12] A. Isacsson, M.-C. Cha, K. Sengupta, and S. M. Girvin, Phys. Rev. B 72, 184507 (2005).

[13] E. Altman, E. Demler, and M. D. Lukin, Phys. Rev. A 70, 013603 (2004).

[14] J. Simon, W. S. Bakr, R. Ma, M. E. Tai, P. M. Preiss, and M. Greiner, Nature 472, 307 (2011).

[15] A. Polkovnikov, K. Sengupta, A. Silva, and M. Vengalattore, eprint: arXiv.org:1007.5331 to appear in Rev. Mod. Phys..

[16] J. Dalibard, F. Gerbier, G. Juzeliūnas, and P. Öhberg, eprint: arXiv.org:1008.5378.

[17] D. Jaksch and P. Zoller, New J. Phys. 5, 56 (2003).

[18] G. Juzeliūnas and P. Öhberg, Phys. Rev. Lett. 93, 033602 (2004)

[19] I. I. Satija, D. C. Dakin, and C. W. Clark, Phys. Rev. Lett. 97, 216401 (2006)
[20] H. Zhai, R. O. Umucalılar, and M. Ö. Oktel, Phys. Rev. Lett. 104, 145301 (2010).

[21] S. L. Zhu, H. Fu, C. J. Wu, S. C. Zhang, and L. M. Duan, Phys. Rev. Lett. 97, 240401 (2006).

[22] Y.-J. Lin, R. L. Compton, K. Jiménez-García, J. V. Porto, and I. B. Spielman, Nature 462, 628 (2009).

[23] Y.-J. Lin, R. L. Compton, A. R. Perry, W. D. Phillips, J. V. Porto, and I. B. Spielman, Phys. Rev. Lett. 102, 130401 (2009)

[24] S. Powell, R. Barnett, R. Sensarma, and S. Das Sarma, Phys. Rev. Lett. 104, 255303 (2010)

[25] S. Sinha and K. Sengupta, Europhys. Lett. 93, 30005 (2011)

[26] K. Saha, K. Sengupta, and K. Ray, Phys. Rev. B 82, 205126 (2010).

[27] K. Osterloh, M. Baig, L. Santos, P. Zoller, and M. Lewenstein, Phys. Rev. Lett. 95, 010403 (2005).

[28] J. Ruseckas, G. Juzeliūnas, P. Öhberg, and M. Fleischhauer, Phys. Rev. Lett. 95, 010404 (2005).

[29] N. Goldman, A. Kubasiak, P. Gaspard, and M. Lewenstein, Phys. Rev. A 79, 023624 (2009).

[30] F. E. A. dos Santos and A. Pelster, Phys. Rev. A 79, 013614 (2009).

[31] B. Bradlyn, F. E. A. dos Santos, and A. Pelster, Phys. Rev. A 79, 013615 (2009).

[32] P. Pérez-Fernández, P. Cejnar, J. M. Arias, J. Dukelsky, J. E. García-Ramos, and A. Relaño, Phys. Rev. A 83, $033802(2011)$

[33] N. Lindner, A. Auerbach, and D. P. Arovas, Phys. Rev. B 82, 134510 (2010)

[34] K. V. Krutitsky and R. Graham, Phys. Rev. A 70, 063610 (2004)

[35] N. Goldman, A. Kubasiak, A. Bermudez, P. Gaspard, M. Lewenstein, and M. A. Martin-Delgado, Phys. Rev. Lett. 103, 035301 (2009).

[36] T. Grass, M. A. Baranov, and M. Lewenstein, eprint: arXiv .org: 1105. 0299.

[37] D. R. Hofstadter, Phys. Rev. B 14, 2239 (1976). 
[38] K. Byczuk and D. Vollhardt, Phys. Rev. B 77, 235106 (2008).

[39] L. Balents, L. Bartosch, A. Burkov, S. Sachdev, and K. Sengupta, Phys. Rev. B 71, 144508 (2005). 